\title{
Infant regulatory function acts as a protective factor for later traits of autism spectrum disorder and attention deficit/ hyperactivity disorder but not callous unemotional traits
}

Rachael Bedford ${ }^{1 *}$ (D), Teodora Gliga ${ }^{2}$, Alexandra Hendry ${ }^{3}$, Emily J. H. Jones ${ }^{2}$, Greg Pasco ${ }^{4}$, Tony Charman ${ }^{4}$, Mark H. Johnson ${ }^{2,5}$, Andrew Pickles ${ }^{1}$ and The BASIS Team

\begin{abstract}
Background: Reduced executive functions (EF) are commonly associated with developmental conditions (e.g., autism spectrum disorder, ASD; attention deficit/hyperactivity disorder, ADHD), although EF seems to be typical in children with callous unemotional (CU) traits. Regulatory function (RF) is a proposed infant precursor that maps on onto factors driving later EF. Here, we first test whether RF is specifically and negatively associated with ASD and ADHD traits, but not CU traits. Second, we test whether RF can act as a protective factor, by moderating the association between infant markers and subsequent ASD and ADHD traits.

Methods: Participants were 79 infants at high $(N=42)$ and low $(N=37)$ familial risk for ASD. Data come from the 14-month infant visit (Autism Observational Scale for Infants; AOSI; activity level and RF from the Infant Behavior Questionnaire; IBQ) and the 7-year visit (ASD traits: Social Responsiveness Scale, SRS; ADHD traits: Conners 3, CU traits: Inventory of Callous Unemotional Traits).

Results: Infant RF was negatively associated with later traits of ASD $(B=-0.5, p=0.01)$ and ADHD inattention $(B=-0.24, p=0.02)$ but not hyperactivity $(B=-0.25, p=0.10)$ or $C U$ traits $(B=0.02, p=0.86)$. RF moderated the association between infant AOSI score and ASD traits, with a significant effect in those with low RF $(B=0.10$, $p=0.006)$, not high RF $(B=0.01, p=0.78)$. Similarly, for $A D H D$, infant activity level was associated with later ADHD inattention in those with low $(B=0.17, p=0.04)$ but not high $\operatorname{RF}(B=0.07, p=0.48)$. For $A D H D$ hyperactivity symptoms, activity level was predictive at both high and low levels of RF.

Conclusions: Strong RF may allow children to compensate for other atypicalities, thus attenuating the association between infant markers and later disorder traits. Whilst infant RF was associated with both ASD and ADHD inattention traits, there was no association with ADHD hyperactivity or CU traits. This suggests that any protective effect may not be universal and emphasises the need for a better understanding of the underlying moderating mechanisms.
\end{abstract}

Keywords: Autism spectrum disorder, Attention deficit/hyperactivity disorder, Callous unemotional traits, Executive function, Infants at risk, Regulatory function

\footnotetext{
* Correspondence: rachael.bedford@kcl.ac.uk

${ }^{1}$ Biostatistics and Health Informatics Department, Institute of Psychiatry,

Psychology \& Neuroscience, King's College London, London, UK

Full list of author information is available at the end of the article
}

(c) The Author(s). 2019 Open Access This article is distributed under the terms of the Creative Commons Attribution 4.0 International License (http://creativecommons.org/licenses/by/4.0/), which permits unrestricted use, distribution, and reproduction in any medium, provided you give appropriate credit to the original author(s) and the source, provide a link to the Creative Commons license, and indicate if changes were made. The Creative Commons Public Domain Dedication waiver (http://creativecommons.org/publicdomain/zero/1.0/) applies to the data made available in this article, unless otherwise stated. 


\section{Background}

Executive functions (EF) refer to a set of skills including planning, online monitoring, inhibition and working memory, which support the ability to set and achieve goals [1]. EF difficulties are associated with a broad range of acquired and developmental disorders [2], including autism spectrum disorder (ASD [3]) and attention deficit/hyperactivity disorder (ADHD [4]). Johnson [5] argues that the co-occurrence between poor EF and some neurodevelopmental disorders could arise because young children with poor EF skills are less able to adapt to, or compensate for, atypicalities in other brain systems early in life. In typical development, EF emerges across a protracted period and is commonly only measured experimentally from around preschool age. Thus, in order to test for potential moderating effects of EF earlier in life, it is necessary to identify precursors to EF during infancy. One such proposed precursor [6] is infants' regulatory function $(\mathrm{RF})$, a set of processes that modulate an individual's response/reactivity to a change in their environment.

Children with autism spectrum disorder (ASD) show high levels of co-occurring conditions including ADHD [7] and callous unemotional (CU) traits (Leno et al. 2015). In order to tease apart shared and distinct pathways, it is helpful to compare conditions with and without shared genetic overlap. There is high co-occurrence and overlapping genetic risk between ASD and attention deficit/hyperactivity disorder (ADHD [7-9]). Indeed, this has been taken by some to suggest a common developmental pathway [10]. Whilst children with high callous unemotional traits show some superficial phenotypic overlap with ASD, particularly in social-affective skills such as emotion recognition, the disorders have separate genetic aetiology [11]. This suggests different underlying developmental pathways. The current paper aims to test two key hypotheses. First, are atypicalities in infant RF specific to traits of particular disorders (i.e., ASD and ADHD), rather than a common shared factor? Second, does infant RF moderate the association between known infant markers and later disorder traits?

Difficulties in EF have been extensively observed amongst children and adults with autism spectrum disorder (ASD) [3], particularly for cognitive flexibility and the ability to co-ordinate multiple EF demands simultaneously [12]. Whilst EF is no longer considered likely to be the only causal mechanism involved in ASD, it may act as a modifier of the phenotype, which interacts with atypicalities in core domains such as social cognition, exacerbating or lessening symptom expression $[5,13]$. ADHD has been linked most consistently to difficulties with inhibitory control, and the co-occurrence of ADHD symptoms in ASD is associated with lower performance on inhibition tasks $[12,14]$. As with ASD, there is debate in the literature about the extent to which EF difficulties represent a core component of the disorder rather than a co-occurring component [15].

Whilst associated EF deficits are indeed common across different developmental disorders, they may not be universally affected in atypical development. Children with high callous unemotional (CU) traits are characterised by a lack of guilt, remorse and empathy; traits thought to map onto the construct of psychopathy $[16,17]$. Very limited research has specifically investigated EF in individuals with high $\mathrm{CU}$ traits. Amongst children with ASD, who overall show difficulties with conflict monitoring [18], those with higher co-occurring $\mathrm{CU}$ traits are associated with relatively superior conflict monitoring [19]. Other studies show no difference in executive function performance between autistic children and adolescents with high versus low co-occurring CU traits [20, 21]. In the current paper, we test whether an infant precursor to later EF is associated specifically with ASD and ADHD traits, but not $\mathrm{CU}$ traits, in a population that is at increased risk for manifesting traits of all three of these conditions.

In order to test the associations between an infant precursor to EF and later emerging traits of developmental disorders, the current study uses a longitudinal, prospective sample of infants at familial risk for ASD. An estimated $20 \%$ of infants at high familial risk for ASD (who have an older sibling with an ASD diagnosis) go on to a clinical diagnosis of ASD themselves (e.g., [22]) and a further $20 \%$ show other developmental atypicalities: subclinical symptoms of ASD (referred to as the broader autism phenotype (BAP), low IQ scores, and symptoms of co-occurring disorders such as ADHD [23, 24]. At-risk sibling designs support the investigation of both clinical and subclinical (e.g., BAP) phenotypes of ASD; this may yield important insights into risk and protective mechanisms that are not afforded by classic case-control designs [25]. Further, prospective infant-sibling designs may reveal early differences that are later masked or complicated by intellectual ability, compensatory or secondary mechanisms, and interactions with co-occurring symptoms [26].

Hendry et al. [6] argue that infant RF maps onto factors driving child and adolescent EF. RF is captured by the regulatory capacity/orienting (RCO) composite from the Infant Behavior Questionnaire-Revised (IBQ-R) [27] and/or the effortful control composite of the Early Childhood Behavior Questionnaire [28] with toddlers. RCO measures infants' ability to sustain and shift attention depending on environmental needs, their enjoyment of novelty and ability to recover from distress and their enjoyment of social closedness. From late infancy and beyond, RF is largely self-directed, but in the first year of life, RF is at least partially contingent on caregiver actions (i.e., an adult providing the means of distraction or offering a soothing cuddle) [29]. An important index of 
$\mathrm{RF}$ is thus the infant's response to parent soothing. In toddlers, the effortful control composite of the Early Childhood Behavior Questionnaire [28] also captures the ability to plan and execute actions. Scores on the RCO composite in infancy predict effortful control scores at $18-22$ months $[30,31]$ and $2-3$ years of age $[32,33]$. In turn, by the end of the second year of life, effortful control shows measurement and conceptual overlap with measures of EF [6].

Several studies have shown links between lower infant and toddler effortful control, including inhibitory control and later ADHD symptoms [34-36]. Similarly, in relation to later ASD outcome, previous infant-sibling studies have demonstrated that low parent-reported RF and effortful control are predictive of risk group membership and later ASD diagnosis from as early as 14 months $[37,38]$. In line with this developmental timing, the executive attention network, which regulates orienting of attention and information processing, is thought to gain functionality towards the end of the first year of life [6,39]. In order to understand the potential moderating effects of infant RF, here, we aim to test whether infant RF can moderate the association between known infant markers, which have previously been associated with ASD and ADHD traits.

Several early markers for ASD in infancy have been identified [40], which are precursors later symptomatology. Here, we chose a global measure of early autismlike behaviours, the Autism Observation Scale for Infants (AOSI [41]). This observational assessment measures behaviours including imitation, motor skills and the anticipation of social interaction. AOSI total scores measured when infants at-risk for ASD are aged 1 year are associated with later ASD symptoms and diagnostic outcome [42] and have previously been shown to be predictive of ASD outcome in the current cohort [43]. For ADHD, fewer infant markers have been identified, but one which may be specific to ADHD (as compared to ASD) in the current cohort is activity level [36] measured by the Infant Behavior Questionnaire-Revised (IBQ-R) [27]. Testing the effect of the interaction between AOSI scores and infant RF on later ASD symptoms and of the interaction between activity level and RF on later ADHD traits will enable us to test whether high RF has broad protective effects in relation to later outcome. Early markers of $\mathrm{CU}$ traits are less well established, and although some putative markers have previously been suggested (e.g., reduced face preference in 5-week-olds [44], and increased fearfulness in 14-month-olds [45]), these measures were not collected in the current cohort, and their specificity to later $\mathrm{CU}$ traits is also unknown.

In the current study, we first test the hypothesis that infant RF will be negatively associated with traits of ASD and ADHD, but not CU traits, predicting a significant difference in the strength of association between ASD,
ADHD and CU traits. Second, we test the hypothesis that RF moderates the association between known infant marker (activity level for ADHD, and AOSI early autism-like behaviours for ASD) with later traits of ASD and ADHD [5].

\section{Method \\ Participants}

As part of the British Autism Study of Infant Siblings (BASIS: www.basisnetwork.org), 104 infants (54 highrisk, 21 male; 50 low-risk, 21 male) took part in a battery of assessments at 7 and 14 months and 2, 3 and 7 years. At enrolment, each high-risk (HR) infant ( $n=$ 54) had an older sibling (in 4 cases, a half-sibling) with a community clinical ASD diagnosis, confirmed using information from the Development and WellBeing Assessment (DAWBA [46]) and the Social Communication Questionnaire (SCQ [47]) by expert clinicians on our team $(\mathrm{TC}, \mathrm{PB})^{1}$. Low-risk (LR) controls $(n=50)$ were full-term infants (with one exception) recruited from a volunteer database at the Birkbeck Centre for Brain and Cognitive Development. For older siblings of LR infants, the SCQ was used to confirm the absence of ASD, with no child scoring above instrument cut-off ( $\geq 15 ; n=1$ missing data).

Of $53 \mathrm{HR}$ and $48 \mathrm{LR}$ children retained at the 3-year assessment, 44 HR (83\%) and $37 \mathrm{LR}(77 \%)$ agreed to take part in the follow-up study at 6-8 years. Of these, two HR children did not complete a research visit (parents completed questionnaires only). The retained sample did not differ from the non-retained sample in 3-year levels of ASD on the Autism Diagnostic Observation Schedule-Generic (ADOS-G [48]), Social Responsiveness Scale-Second Edition (SRS-2 [49]) or SCQ, developmental level on the Mullen Scales of Early Learning [50], adaptive behaviour assessed with the Vineland Adaptive Behavior Scales-Second Edition (VABS-II [51]), or family income (all $p>.4$ ). The HR and LR groups did not differ in age (HR mean (SD), 90.8 (6.3) months; LR mean (SD), 89.3 (4.8) months; $t(76)=-1.13, p=.26)$ or sex (HR, 36.4\% male; LR, $40.5 \%$ male; $\chi^{2}(1)=.15, p=.70$ ) at the follow-up. The exact sample size differs between analyses depending on the missingness of independent variables, with $N=69$ for the ASD regression model $(\mathrm{HR}=36 ; \mathrm{LR}=33)$ and $N=76$ for the ADHD models $(\mathrm{HR}=41 ; \mathrm{LR}=35)$. Ethical approval was obtained from the NHS National Research Ethics Service (NHS RES London REC 08/H0718/76; 14/LO/0170). Parents provided written informed consent. At the mid-childhood visit, children provided written informed assent wherever possible given the developmental level.

\footnotetext{
${ }^{1} 5$ DAWBA and 5 SCQ missing.
} 


\section{ASD outcome at 7 years of age (see [52] for full sample description)}

To ascertain ASD diagnostic outcome according to DSM5 , four experienced researchers (ES, BM, GP, TC) reviewed information on ASD symptomatology (Autism Diagnostic Observation Schedule-Second Edition, ADOS-2, [53] Autism Diagnostic Interview-Revised ADI-R, [54] SCQ, for HR participants only) and adaptive functioning (VABSII) and IQ (Wechsler Abbreviated Scale of Intelligence-Second Edition, WASI-II, [55]) for every HR and LR child. Fifteen HR children (7 boys, 8 girls) met DSM-5 (APA, 2013) criteria for ASD at age 7, and the remaining 27 HR children ( 8 boys, 19 girls) did not. The 2 HR children who completed only questionnaires were not categorised. None of the 37 LR children met the DSM-5 criteria for ASD, and none had a community clinical ASD diagnosis at 7 years. Group characteristics at age 7 are presented in Additional file 1: Table S1.

\section{Fourteen-month infant measures}

The Autism Observation Scale for Infants (AOSI [41, 43]) is a semi-structured observational assessment of ASD behavioural markers in infancy collected at 7 and 14 months. In the current study, a 19-item version of the AOSI was used (see [56]) with items coded $0,1,2$ or 3, which gives a total score (sum of all codes), with higher scores indexing greater atypicality. The majority of assessments were double coded with excellent reliability $(n=85$, intraclass correlation coefficient $=0.95)$.

\section{Infant Behavior Questionnaire-Revised (IBQ-R) [27]}

The IBQ-R (191 items) is a measure of temperament in which parents rate the frequency with which their infant exhibits particular behaviours in everyday contexts on a seven-point scale (never to always). Parents rate their child's behaviour over the past week (IBQ-R). Data for the present analysis comes from the 14-month visit. Whilst Rothbart and colleagues conventionally recommend the use of the ECBQ for infants aged 13-17 months, they note that the IBQ may be more appropriate for use with samples with potential developmental delays [57]. IBQ-R items are averaged to yield subscales indexing different temperament dimensions, which show high continuity across instruments [33]. In the current study, regulatory function $(R F)$ was chosen as an infant precursor to later emerging executive functions. Our measure of RF is the orienting/regulation factor subscale of the IBQ-which is a composite score of the duration of orienting, low intensity pleasure, cuddliness and soothability scales [27].

Activity level from the IBQ- $\mathrm{R}$ was also used as an infant marker for later emerging ADHD symptoms [36]. The activity subscale assesses limb movement, squirming and locomotor activity. Note that the items comprising the activity subscale are entirely different from those items used to assess RF (i.e., duration of orienting, low intensity pleasure, cuddliness and soothability scales).

\section{Seven-year outcome measures \\ Social Responsiveness Scale-2 (SRS-2 [49])}

A parent-report measure of ASD symptoms (rated over the 6 months prior to testing), the SRS-2 was chosen as the measure of ASD traits in the current analysis to be comparable with the parent-reported ADHD and CU trait measures. Age-normed SRS-2 T-scores were used (mean 50; SD 10; minimum-maximum $\leq 30$ to $\geq 90$ ).

\section{Conners 3 ([58])}

The parent-rated Conners 3 was used to measure symptoms of ADHD (also rated over the 6 months before testing). T-scores (mean 50; SD 10; minimum-maximum score $\leq 30$ to $\geq 90$ ) for the inattention and hyperactivity/ impulsivity domains were used in analyses.

\section{Inventory of Callous Unemotional Traits (ICU [59])}

The ICU is a parent report questionnaire with 24 items assessing uncaring, callous and unemotional behaviours. Each item is rated on a 4-point Likert scale from 0 (not at all true) to 3 (definitely true). The total score is used in the analysis.

\section{Statistical analysis}

Descriptive statistics are presented in Tables 1 and 2 . Analyses were performed in Stata [60]. The outcome variables-SRS T-score, Conners ADHD inattention and hyperactivity T-scores and ICU scores-were not normally distributed and were transformed using a lnskew0 transformation in Stata, which normalised all scales (Shapiro-Wilk test $p$ values $>0.11$ ). For regression models with interaction terms, all variables were mean centred.

First, a multivariate regression model was run to test whether RF was associated with ASD and ADHD (hyperactivity and inattention) but not CU traits. The 'test' command in Stata was used to run pairwise coefficient comparisons. Next, to test for a moderating role of RF, we ran multiple regressions in Stata. In the ASD regression model, we tested whether ASD symptoms (7year SRS score) were predicted by 14-month AOSI score, 14 months RF, and their interaction, controlling for group (high versus low familial risk). In the ADHD models, we tested whether hyperactivity and inattention were predicted by 14-month activity, RF, and their interaction, again controlling for risk group. Because we hypothesised that the infant marker will be associated with later disorder traits only for those with low RF, we also planned to run separate regressions to test the association in those with high and low RF separately, again controlling for risk group. 
Table 1 Infant risk and protective markers at 14 months: AOSI score, activity level and regulatory function

\begin{tabular}{llll}
\hline & High-risk & Low-risk & Overall \\
\hline Early ASD-like behaviour (AOSI) & & \\
Mean (SD) & $4.88(4.52)$ & $3.31(3.57)$ & $4.14(4.15)$ \\
$N$ & 41 & 36 & 77 \\
Activity level (IBQ-R) & & \\
Mean (SD) & $4.27(0.96)$ & $4.010(0.74)$ & $4.19(0.86)$ \\
$N$ & 41 & 35 & 76 \\
Regulatory function (IBQ-R) & & \\
Mean (SD) & $4.47(0.65)$ & $4.81(0.47)$ & $4.63(0.60)$ \\
$N$ & 41 & 35 & 76 \\
\hline
\end{tabular}

AOSI Autism Observation Scale for Infants, IBQ- $R$ Infant Behavior Questionnaire-revised

Table 2 shows the mean trait scores for ASD, ADHD and $\mathrm{CU}$ traits split by risk group. Correlations with the centred, skew-transformed trait scores show ASD traits were significantly positively correlated with both ADHD inattention and hyperactivity $(r=0.42, p<0.001 ; r=0.46$, $p<0.001)$ and $\mathrm{CU}$ traits $(r=0.42, p<0.001)$.

Additional analyses are included in Additional file 1: Tables S2a, b, S3 and S4a-c: (1) correlations between RF and subdomains of ASD (social communication and RRBs) and CU traits (callous, unemotional, uncaring); (2) regression models re-run with Social Communication Questionnaire (SCQ) score as the outcome measure, because the SRS has been shown to relate to domain-general difficulties as well as ASD severity [61]; and (3) regression models which do not covary for risk group. Results from these additional and confirmatory analyses remain substantively similar.

Table 2 ASD, ADHD and CU traits at 7 years

\begin{tabular}{|c|c|c|c|}
\hline & High-risk & Low-risk & Overall \\
\hline \multicolumn{4}{|c|}{ ASD traits (SRS-2) } \\
\hline Mean (SD) & $59.27(19.63)$ & $45.49(5.82)$ & $52.57(16.11)$ \\
\hline N & 37 & 35 & 72 \\
\hline \multicolumn{4}{|c|}{ ADHD inattention (Conners 3) } \\
\hline Mean (SD) & $57.07(13.95)$ & $51.22(9.40)$ & $54.33(12.32)$ \\
\hline N & 42 & 37 & 79 \\
\hline \multicolumn{4}{|c|}{ ADHD hyperactivity (Conners 3) } \\
\hline Mean (SD) & $59.26(16.59)$ & $52.16(11.58)$ & $55.94(14.81)$ \\
\hline N & 42 & 37 & 79 \\
\hline \multicolumn{4}{|c|}{ Callous unemotional traits (ICU) } \\
\hline Mean (SD) & $21.83(11.27)$ & $18.09(5.96)$ & $20.17(9.4)$ \\
\hline$N$ & 40 & 32 & 72 \\
\hline
\end{tabular}

\section{Results}

Regulatory function is associated with ASD and ADHD but not CU traits

A multivariate regression model showed a significant association between 14-month RF and later 7-year traits of ASD $(b=-0.497, \quad$ S.E. $=0.197, p=0.014)$, ADHD inattention $(b=-0.239$, S.E. $=0.101, p=0.021)$, though not hyperactivity $(b=-0.248$, S.E. $=0.151, p=$ 0.104). No significant association was found between RF and later CU traits $(b=0.016$, S.E. $=0.089, p=0.861)$. Testing the difference between slopes showed that the association between $\mathrm{RF}$ and $\mathrm{CU}$ traits was significantly different to that for RF with ASD $(p=0.005)$ and ADHD inattention $(p=0.027)$, but not different to ADHD hyperactivity $(p=0.096)$.

Does RF moderate the association between infant autismlike behaviours and later ASD symptoms at 7 years?

A multiple regression showed significant associations between 7-year SRS score and risk group $(b=0.799$, S.E $=$ $0.212, p<0.001)$ and age $(b=-0.995, \mathrm{~S} . \mathrm{E}=0.300, p=$ $0.002)$, with no main effect of sex $(b=0.024$, S.E $=0.219$ $p=0.914)$. There was no main effect of RF $(b=-0.215$, $\mathrm{S} . \mathrm{E}=0.176, p=0.227)$, and the association with 14month AOSI did not reach significance $(b=0.045$, S.E $=$ $0.023 p=0.056)$. However, the interaction between AOSI and RF was significant $(b=-0.107, \mathrm{~S} . \mathrm{E}=0.048, p=$ 0.031). To break this down, we ran regressions separately for high and low RF (based on a median split; see Fig. 1a, b), whilst controlling for risk group, age and sex. The AOSI was a significant predictor of SRS in those with low RF ( $b=0.103$, S.E $=0.034, p=0.006)$ over and above the effect of risk group $(b=0.870$, S.E $=0.256, p=0.002)$ and age $(b=-0.783, \mathrm{~S} . \mathrm{E}=0.330, p=0.025)$; the main effect of sex was not significant $(b=0.082$, S.E $=0.268, p=$ $0.761)$. There was no association for those with high RF $(b=0.009, \mathrm{~S} . \mathrm{E}=0.033, p=0.776)$. Risk group and age were both significant $(b=0.721, \mathrm{~S} . \mathrm{E}=0.313, p=0.028 ; b=-$ 1.328 , S.E $=0.525, p=0.017$, respectively) and sex was not $(b=0.144, \mathrm{~S} . \mathrm{E}=0.331, p=0.667)$.

\section{Does $\mathrm{RF}$ moderate the association between infant activity} level and later ADHD symptoms at 7 years?

For ADHD inattention, a multiple regression showed no significant associations between 7-year inattention score and either risk group $(b=0.193, \mathrm{~S} . \mathrm{E}=0.111, p=0.087)$, age $(b=-0.199, \mathrm{~S} . \mathrm{E}=0.162, p=0.225)$ or sex $(b=0.153$, $\mathrm{S} . \mathrm{E}=0.121, p=0.210)$. The main effect of RF $(b=-0.201$, $\mathrm{S} . \mathrm{E}=0.108, p=0.067)$ and 14 -month activity $(b=0.126$, $\mathrm{S} . \mathrm{E}=0.064, p=0.054)$ did not reach significance. There was no significant interaction between activity and RF ( $b=0.103$, S.E $=0.077, p=0.188)$. Although the interaction was not significant, as we had a specific a priori hypothesis about the association between activity score and ADHD 

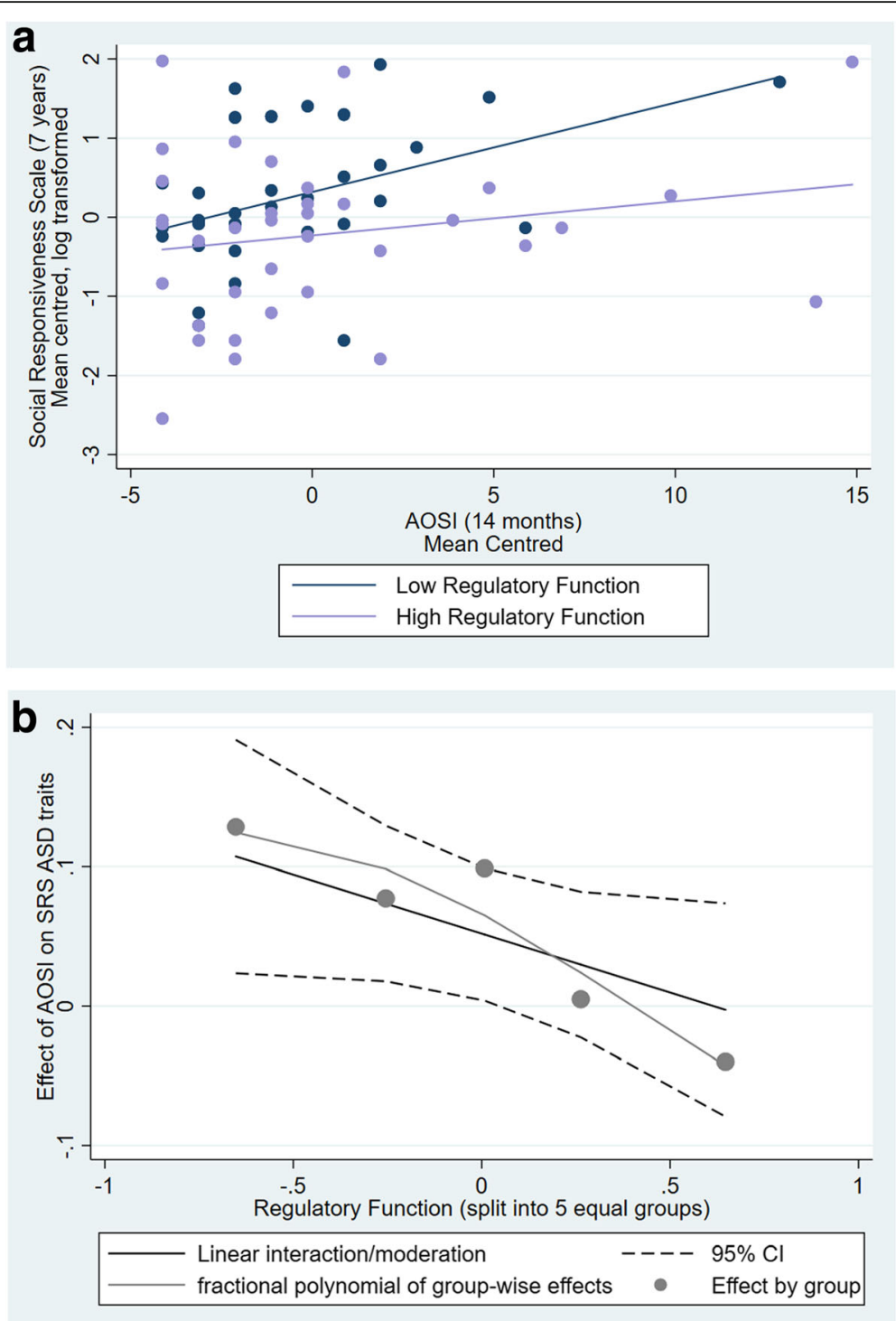

Fig. 1 a The effect of infant AOSI score predicting later ASD traits on the SRS for those with high versus low RF. b The effect of RF (split into 5 equal groups) on the association between AOSI and SRS scores. At higher levels of RF, there is no association between AOSI and SRS, but at low RF levels, AOSI and SRS scores are positively associated

traits depending on RF level, we ran the regressions separately for high and low RF. Activity was a significant predictor of inattention for those with low RF $(b=0.171$, $\mathrm{S} . \mathrm{E}=0.081, p=0.043$, see Fig. 2) controlling for risk group $(b=0.138$, S.E $=0.157, p=0.387)$, age $(b=-0.182$, S.E $=$ $0.207, p=0.386)$ and $\operatorname{sex}(b=0.125$, S.E $=0.159, p=0.436)$. There was no association between activity and ADHD inattention for those with high RF $(b=0.069$, S.E $=0.096$, $p=0.477)$, and no significant effect of risk group $(b=$ 0.209 , S.E $=0.151, p=0.175)$, age $(b=-0.296$, S.E $=0.259$, $p=0.262)$ and sex $(b=0.129$, S.E $=0.173, p=0.463)$.

For ADHD hyperactivity, a multiple regression showed no significant associations between 7-year hyperactivity score and either risk group $(b=0.251$, S.E $=0.153, p=$
0.106), age $(b=-0.377, \mathrm{~S} . \mathrm{E}=0.224, p=0.097)$ or $\operatorname{sex}(b=$ 0.251 , S.E $=0.167, p=0.137)$. The main effect of RF was not significant $(b=-0.084$, S.E $=0.149, p=0.573)$, but there was a significant association with 14-month activity $(b=$ 0.387 , S.E $=0.089, p<0.001)$. There was no significant interaction between activity and RF $(b=-0.024$, S.E $=0.107$, $p=0.826$ ). When this was broken down by RF, for children with low RF, activity was a significant predictor of hyperactivity $(b=0.452$, S.E $=0.126, p=0.001)$ with no significant effect of risk group $(b=0.396$, S.E $=0.243, p=0.114)$, age $(b=-0.409, \mathrm{~S} . \mathrm{E}=0.321, p=0.211)$ and sex $(b=0.238$, $\mathrm{S} . \mathrm{E}=0.247, p=0.343)$. Results were similar for high RF, with activity $(b=0.309, \mathrm{~S} . \mathrm{E}=0.119, p=0.014)$ and not risk group $(b=0.094, \mathrm{~S} . \mathrm{E}=0.187, p=0.617)$, age $(b=-0.449$, 


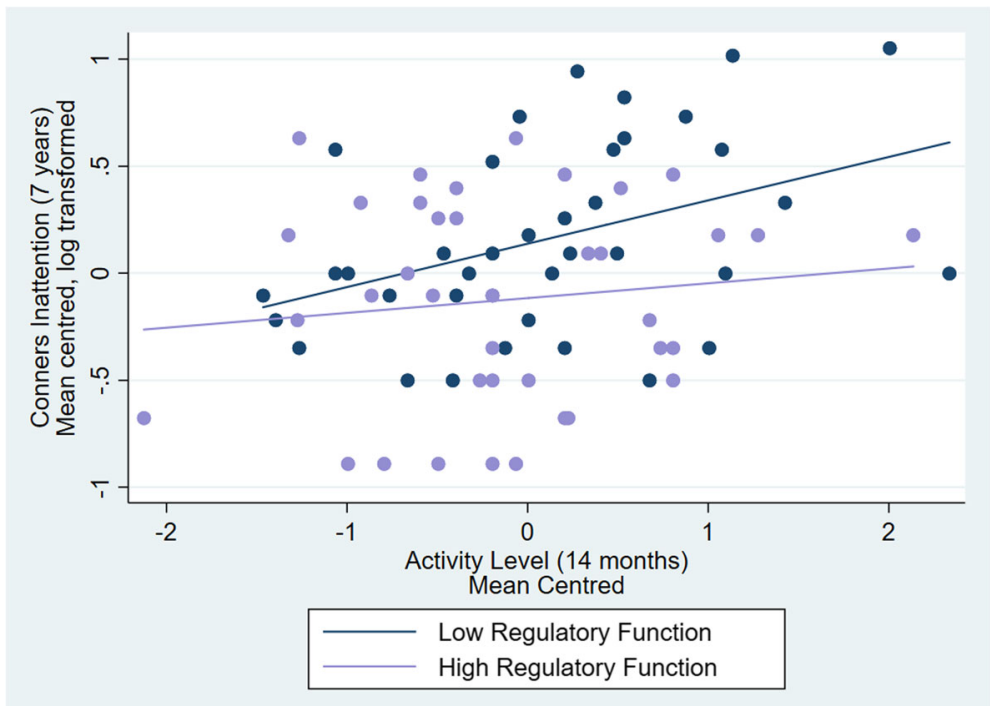

Fig. 2 The effect of infant activity level predicting later ADHD inattention traits on the Conners for those with high versus low RF

S.E $=0.320, p=0.170)$ or $\operatorname{sex}(b=0.299$, S.E $=0.214, p=$ 0.172 ) associated with later ADHD hyperactivity.

\section{Discussion}

In line with the hypotheses presented earlier, we found evidence for a differential association between infant regulatory function $(\mathrm{RF})$ and later disorder traits, with reduced RF significantly associated with later ASD and ADHD traits, but not with $\mathrm{CU}$ traits. Further, consistent with predictions from Johnson [5], we found evidence to support a protective role of strong RF within ASD, and to a lesser extent, ADHD traits: infant autism-like atypicality as measured by the AOSI was associated with later 7-year ASD traits only in those with low RF as infants. A similar effect was found for ADHD inattention traits; infant activity level was associated with later ADHD inattention only in those with low RF. However, for ADHD hyperactivity, activity level was significantly associated with later hyperactivity traits in both those with high and low EF.

The association between 14-month infant regulation and later traits of ASD at 7 years extends previous findings in the same sample in relation to 3-year ASD outcome [37]. Clifford et al. [37] found that children who went on to an ASD diagnosis at 3 years had lower levels of regulation (termed effortful control in their paper as it was measured at both 14 months and later in development at 24 months, when inhibitory control is included in the subscale). Whilst there is a good degree of stability in clinical diagnosis from toddlerhood to mid-childhood in infants at risk for ASD $[52,62]$, there is nevertheless still change with some children only meeting diagnostic criteria at a later age and others meeting criteria as toddlers but not in mid- childhood. It is useful to know whether the same infant markers remain predictive later in development (e.g., [63]), and future studies with larger samples should test whether infant predictors such as regulation abilities can discriminate stable cases from those who shift diagnostic categories or show a great degree of change in symptom severity.

We also found a significant association between regulatory function and traits of ADHD inattention, suggesting that this effect is not ASD specific. For ADHD hyperactivity, however, the correlation with early RF did not reach significance (although it is worth noting that the beta was the same as for inattention, so this will need to be replicated in larger samples). These findings are consistent with previous studies showing that the hyperactive-impulsive subtype of ADHD is not strongly associated with EF difficulties (e.g., $[64,65])$. Indeed, Willcutt et al. [4] in a review of the literature suggested that EF weaknesses may be associated predominately with inattention symptoms.

In contrast to ASD and ADHD inattention, no association was found between infant RF and later $\mathrm{CU}$ traits. The difference in the association between RF and later CU traits compared to both ASD and ADHD inattention traits approached significance. This is consistent with previous findings that, unlike in other developmental disorders [2], EFs do not appear to be impaired in psychopathic or CU traits $[19,66,67]$. This may be because there are differential associations between different subtypes of $\mathrm{CU}$, with impaired $\mathrm{EF}$ in those children who show a profile of $\mathrm{CU}$ traits with high anxiety, more akin to secondary psychopathy, but enhanced EF in those with primary psychopathy traits [68]. In future studies, characterising the specific associations between EF and different 'constructs' [69] such as attention and social 
communication, rather than just the clinical disorders or symptoms, will be important. This will allow us to make specific predictions about how the presence of a cooccurring disorder, with a different profile of strengths and weaknesses, might change performance for a given construct or task (e.g., $[19,70])$.

Our second aim, following the hypothesis put forward by Johnson [5], was to test whether high executive functions-or in this case regulatory functions, as a precursor of executive function-could act as a protective factor. We found evidence that early behavioural signs measured by the AOSI were only associated with later ASD symptoms in those with low RF. Johnson [5] suggests that strong EF may play a protective role by modulating neural activity according to the capacity of posterior brain regions, i.e., selecting the best combination of computational regions for a given learning problem. How might this translate to the behavioural level? One possibility is that it enables more flexible use of compensatory strategies. In ASD, strong RF could help mitigate the impact of early markers (in this case early autism-like behavioural atypicalities) via multiple possible mechanisms. For example, learning and remembering to make eye contact and explicit emotion understanding/theory of mind through logical reasoning [71]. Related to this, strong RF might be better characterised as masking rather than truly reducing autistic traits-it is possible that the lower SRS scores indicate an increased ability to regulate oneself around others. This type of compensation may be 'shallow' which Livingston and Happé [71] describe as being more effortful and prone to break down under stress.

For ADHD traits, infant activity level was associated with later ADHD inattention in those with low but not high RF. Children showing high infant activity levels but also strong EFs may have a greater ability to choose when and what to pay attention to, resulting in lower inattentive traits later in development. For ADHD hyperactivity, on the other hand, there was no evidence of moderation by RF, with activity level predicting later hyperactivity irrespective of RF. This may be because whilst children with high RF are able to control their behaviour at times, for example when concentrating on a task, they continue to manifest hyperactivity at other times, which results in parents forming a global opinion of activity levels being high. To test this hypothesis, it would be important for future studies to use objective measures of activity, such as accelerometer data.

Whilst the current study set out to test a moderating role of EF, based on the hypothesis by Johnson [5], it is important to acknowledge that at both a statistical and conceptual level, it is also possible that rather than enhanced RF acting as a protective factor, low RF is acting as an additional risk factor, compounding the effects of other early markers of atypicality [72]. Future work should test the cross-lagged effect of infant markers and EF across multiple time points, to clarify the temporal order of effects. However, even with a clear temporal ordering of putative risk and protective factors, it is worth noting that any cognitive protective (or risk) factor may represent a precursor to later symptoms which is, therefore, not independent of outcome. Whilst this possibility remains important to consider, we argue that evidence in favour of RF as a protective/risk factor also includes the fact the RF shows cross-domain (i.e., for social communication and restricted and repetitive behaviours, see Additional file 1) and cross-disorder (i.e., ADHD) influences.

The current sample includes both high- and low-risk infants. Whilst the main effect of risk group is included in the analysis, the relatively modest sample size precludes testing more complex models with interactions between group and RF, as well as 3-way group $\times R F \times$ infant marker interactions. It seems likely that the protective role of RF may work differently in those with and without familial ASD risk, and future studies should test this explicitly. Future more highly powered studies should also investigate the potential moderating role of sex. Bedford et al. (2016) [73] demonstrated that whilst there were no significant sex differences in several infant markers for ASD (including the AOSI score used in the current paper), the association between infant markers and disorder traits was moderated by sex; the association was only significant for boys. Whilst the current study controlled for a main effect of sex, future, more highly powered studies should investigate whether apparent protective effects of biological sex could be explained by increased regulatory function in girls [74].

Another limitation is the use of parent report measures for disorder traits, which likely share measurement error and could have been subject to rater bias. However, such 'halo effects', where parents form a global impression of a child and consequently rate them in a similar way across multiple domains, would be more likely to result in similar associations across all disorder traits, which we did not find. Future studies, with larger sample sizes, should use structural equation modelling approaches, such as bifactor models, to attempt to separate out the shared and distinct variance across disorder traits. Whilst an experimenter-led observational measure was used for early infant autism behaviours (AOSI), activity level was also parent rated, from the IBQ-R. It was chosen because it is the only available infant marker (i.e., in 14-month-olds) that has been shown to specifically predict later ADHD traits, but as noted above, future studies should replicate this with objective measures such as accelerometer data.

These results have potentially significant implications when thinking about targeting EF in interventions. 
Executive function difficulties are related to lower adaptive functioning in both ASD [12, 75] and ADHD [76]. Thus, interventions to support the optimal development of EF may have significant benefits across ASD and ADHD, although of course, we demonstrate no causality in the current study. Promisingly, there is some evidence for the potentially modifiable nature of EF following intervention programs (e.g., [77]), particularly early in development when the brain is most plastic [5, 78]. Given that EF interventions may act to improve a variety of symptoms, they have the potential to offer more widespread crossdomain benefits than disorder-specific interventions.

\section{Conclusions}

In conclusion, our findings showed an association between infants' regulation ability and both ASD and ADHD inattention traits in mid-childhood. We also found suggestive evidence in support of the hypothesis that strong regulation abilities (RF; a precursor to executive function) may have a protective effect across developmental disorders [5]. Early markers for ASD (autismlike behaviours) and ADHD inattention (activity level) were only associated with later disorder traits in children with low RF. This suggests that having strong RFs could potentially allow children to compensate for additional neural or behavioural atypicalities. However, this association with later developmental disorder traits was not universal, with no association for later ADHD hyperactivity or CU traits. Future research is needed to establish which additional factors influence when strong EFs are protective and when they are not.

\section{Additional file}

Additional file 1: Supplementary Materials. (DOCX 24 kb)

\section{Abbreviations}

ADHD: Attention deficit/hyperactivity disorder; ADI-R: Autism Diagnostic Interview—Revised; ADOS-2: Autism Diagnostic Observational Schedule-Second Edition; ADOS-G: Autism Diagnostic Observational Schedule-Generic; AOSI: Autism Observation Scale for Infants; ASD: Autism spectrum disorder; BAP: Broader autism phenotype; BASIS: British Autism Study of Infant Siblings; CU: Callous unemotional; DAWBA: Development and Well-Being Assessment; DSM-5: Diagnostic and Statistical Manual 5th edition; EF: Executive function; IBQ-R: Infant Behavior Questionnaire-Revised; ICU: Inventory of Callous Unemotional Traits; RCO: Regulatory capacity/ orienting; RF: Regulatory function; SCQ: Social Communication Questionnaire; SRS-2: Social Responsiveness Scale-Second Edition; VABS-II: Vineland Adaptive Behavior Scales-Second Edition
\end{abstract}

\section{Acknowledgements}

We are very grateful for the important contributions the BASIS families have made towards this study. Thanks to the BASIS team, in alphabetical order: Simon Baron-Cohen, Patrick Bolton, Bosiljka Milosavljevic, Susie Chandler, Mayada Elsabbagh, Janice Fernandes, Holly Garwood, Kristelle Hudry, Elizabeth Shephard, Leslie Tucker, Agnes Volein.

\section{Authors' contributions}

RB contributed to the design of the study, collected and analysed data, wrote and edited the manuscript. TG contributed to the design of the study, collected data, and helped to draft the manuscript. AH contributed to the design of the study and helped to draft the manuscript. EJ contributed to the design of the study and helped to draft the manuscript. GP collected data and helped to draft the manuscript. TC conceived of the study and helped to draft the manuscript. MJ conceived of the study and helped to draft the manuscript. AP conceived of the study and helped to draft the manuscript. All authors read and approved the final manuscript.

\section{Funding}

The research was supported by the BASIS funding consortium led by Autistica www.basisnetwork.org), Autism Speaks, and a UK Medical Research Council Programme Grant (G0701484) to M. H. Johnson. R. Bedford is supported by a Sir Henry Wellcome Postdoctoral Fellowship and King's Prize Fellowship (204823/Z/16/Z). M. H. Johnson and T. Charman received support from the Innovative Medicines Initiative Joint Undertaking under grant agreement no. 115300, resources of which are composed of financial contribution from the European Union's Seventh Framework Programme (FP7/2007-2013) and EFPIA companies' in-kind contribution. This work was additionally supported by the National Institute for Health Research (NIHR) Mental Health Biomedical Research Centre at South London and Maudsley NHS Foundation Trust and King's College London. The views expressed are those of the author(s) and not necessarily those of the NHS, the NIHR or the Department of Health.

\section{Availability of data and materials}

The datasets used and/or analysed during the current study are available from the BASIS study on reasonable request.

\section{Ethics approval and consent to participate}

Ethical approval was obtained from the NHS National Research Ethics Service (NHS RES London REC 08/H0718/76; 14/LO/0170). Parents provided written informed consent. At the mid-childhood visit, children provided written informed assent wherever possible given developmental level.

\section{Consent for publication}

Not applicable

\section{Competing interests}

The authors declare that they have no competing interests.

\section{Author details}

${ }^{1}$ Biostatistics and Health Informatics Department, Institute of Psychiatry, Psychology \& Neuroscience, King's College London, London, UK. ${ }^{2}$ Centre for Brain and Cognitive Development, Birkbeck College, University of London, London, UK. ${ }^{3}$ Experimental Psychology Department, Oxford University, Oxford, UK. ${ }^{4}$ Psychology Department, Institute of Psychiatry, Psychology \& Neuroscience, King's College London, London, UK. ${ }^{5}$ Psychology Department, Cambridge University, Cambridge, UK.

Received: 15 November 2018 Accepted: 8 July 2019

Published online: 18 July 2019

\section{References}

1. Diamond A. Executive functions. Annu Rev Psychol. 2013;64:135-68.

2. Snyder HR, Miyake A, Hankin BL. Advancing understanding of executive function impairments and psychopathology: bridging the gap between clinical and cognitive approaches. Front Psychol. 2015;6:328.

3. Demetriou EA, Lampit A, Quintana DS, Naismith SL, Song YJC, Pye JE, et al. Autism spectrum disorders: a meta-analysis of executive function. Mol Psychiatry. 2017. https://doi.org/10.1038/mp.2017.75.

4. Willcutt EG, Doyle AE, Nigg JT, Faraone SV, Pennington BF. Validity of the executive function theory of attention-deficit/hyperactivity disorder: a metaanalytic review. Biol Psychiatry. 2005;57(11):1336-46.

5. Johnson MH. Executive function and developmental disorders: the flip side of the coin. Trends Cogn Sci. 2012;16(9):454-7.

6. Hendry A, Jones EJ, Charman T. Executive function in the first three years of life: precursors, predictors and patterns. Dev Rev. 2016;42:1-33. 
7. Simonoff E, Pickles A, Charman T, Chandler S, Loucas T, Baird G. Psychiatric disorders in children with autism spectrum disorders: prevalence, comorbidity, and associated factors in a population-derived sample. J Am Acad Child Adolesc Psychiatry. 2008;47(8):921-9.

8. Rommelse NN, Franke B, Geurts HM, Hartman CA, Buitelaar JK. Shared heritability of attention-deficit/hyperactivity disorder and autism spectrum disorder. Eur Child Adolesc Psychiatry. 2010;19(3):281-95.

9. Taylor MJ, Charman T, Robinson EB, Plomin R, Happé F, Asherson P, Ronald A. Developmental associations between traits of autism spectrum disorder and attention deficit hyperactivity disorder: a genetically informative, longitudinal twin study. Psychol Med. 2013;43(8):1735-46.

10. Rommelse NNJ, Geurts HM, Franke B, Buitelaar JK, Hartman CA. A review on cognitive and brain endophenotypes that may be common in autism spectrum disorder and attention-deficit/hyperactivity disorder and facilitate the search for pleiotropic genes. Neurosci Biobehav Rev. 2011;35:1363-96.

11. O'Nions E, Tick B, Rijsdijk F, Happé F, Plomin R, Ronald A, Viding E. Examining the genetic and environmental associations between autistic social and communication deficits and psychopathic callous-unemotional traits. PLoS One. 2015;10(9):e0134331.

12. Wallace GL, Yerys BE, Peng C, Dlugi E, Anthony LG, Kenworthy L. Assessment and treatment of executive function impairments in autism spectrum disorder: an update. Int Rev Res Dev Disabil. 2016;51:85-122.

13. Mundy PC, Henderson HA, Inge AP, Coman DC. The modifier model of autism and social development in higher functioning children. Res Pract Persons Severe Disabl. 2007;32(2):124.

14. Craig F, Margari F, Legrottaglie AR, Palumbi R, De Giambattista C, Margari L. A review of executive function deficits in autism spectrum disorder and attention-deficit/hyperactivity disorder. Neuropsychiatr Dis Treat. 2016;12:1191.

15. Nigg JT, et al. Causal heterogeneity in attention-deficit/ hyperactivity disorder: do we need neuropsychologically impaired subtypes? Biol Psychiatry. 2005;57:1224-30.

16. Cleckley $\mathrm{H}$. The mask of sanity: an attempt to reinterpret the so-called psychopathic personality. 1st ed. Oxford: Oxford University Press; 1941.

17. Hare RD. Hare Psychopathy Checklist-Revised (PCL-R): technical manual. 2nd ed. Toronto: Multi-Health Systems; 2003.

18. Tye C, Asherson P, Ashwood KL, Azadi B, Bolton P, McLoughlin G. Attention and inhibition in children with ASD, ADHD and co-morbid ASD+ ADHD: an event-related potential study. Psychol Med. 2014;44(5):1101-16.

19. Tye C, Bedford R, Asherson P, Ashwood KL, Azadi B, Bolton P, McLoughlin G. Callous-unemotional traits moderate executive function in children with ASD and ADHD: a pilot event-related potential study. Dev Cogn Neurosci. 2017;26:84-90

20. Carter Leno V, Charman T, Pickles A, Jones CR, Baird G, Happe F, Simonoff E. Callous-unemotional traits in adolescents with autism spectrum disorder. $\mathrm{Br}$ J Psychiatry. 2015;207(5):392-9.

21. Rogers J, Viding E, Blair RJ, Frith U, Happe F. Autism spectrum disorder and psychopathy: shared cognitive underpinnings or double hit? Psychol Med. 2006;36:1789-98.

22. Ozonoff S, Young GS, Carter A, Messinger D, Yirmiya N, Zwaigenbaum L, Stone WL. Recurrence risk for autism spectrum disorders: a Baby Siblings Research Consortium study. Pediatrics. 2011;128(3):e488-95.

23. Messinger D, Young GS, Ozonoff S, Dobkins K, Carter A, Zwaigenbaum L, et al. Beyond autism: a baby siblings research consortium study of high-risk children at three years of age. J Am Acad Child Adolesc Psychiatry. 2013;52(3):300-8.

24. Miller M, losif AM, Young GS, Hill M, Phelps Hanzel E, Hutman T, et al. School-age outcomes of infants at risk for autism spectrum disorder. Autism Res. 2016;9(6):632-42.

25. Gliga T, Jones EJ, Bedford R, Charman T, Johnson MH. From early markers to neuro-developmental mechanisms of autism. Dev Rev. 2014;34(3):189-207.

26. Johnson $\mathrm{MH}$, Gliga T, Jones $\mathrm{E}$, Charman T. Annual research review: infant development, autism, and ADHD-early pathways to emerging disorders. J Child Psychol Psychiatry. 2015;56(3):228-47.

27. Gartstein MA, Rothbart MK. Studying infant temperament via the revised infant behavior questionnaire. Infant Behav Dev. 2003;26(1):64-86.

28. Putnam SP, Gartstein MA, Rothbart MK. Measurement of fine-grained aspects of toddler temperament: the early childhood behavior questionnaire. Infant Behav Dev. 2006;29(3):386-401.

29. Rothbart MK, Sheese BE, Rueda MR, Posner MI. Developing mechanisms of self-regulation in early life. Emot Rev. 2011;3(2):207-13.

30. Bridgett DJ, Gartstein MA, Putnam SP, Lance KO, Iddins E, Waits R, et al. Emerging effortful control in toddlerhood: the role of infant orienting/ regulation, maternal effortful control, and maternal time spent in caregiving activities. Infant Behav Dev. 2011;34(1):189-99.

31. Erickson NL, Gartstein MA, Beauchaine TP. Infant predictors of toddler effortful control: a multi-method developmentally sensitive approach. Infant Child Dev. 2017;26(2):e1971.

32. Gartstein MA, Slobodskaya HR, Putnam SP, Kinsht IA. A cross-cultural study of infant temperament: predicting preschool effortful control in the United States of America and Russia. Eur J Dev Psychol. 2009;6(3):337-64.

33. Putnam SP, Rothbart MK, Gartstein MA. Homotypic and heterotypic continuity of fine-grained temperament during infancy, toddlerhood, and early childhood. Infant Child Dev. 2008;17(4):387-405.

34. Auerbach JG, Berger A, Atzaba-Poria N, Arbelle S, Cypin N, Friedman A, Landau R. Temperament at 7, 12, and 25 months in children at familial risk for ADHD. Infant Child Dev. 2008;17(4):321-38.

35. Einziger T, Levi L, Zilberman-Hayun Y, Auerbach JG, Atzaba-Poria N, Arbelle S, Berger A. Predicting ADHD symptoms in adolescence from early childhood temperament traits. J Abnorm Child Psychol. 2018;46(2): 265-76.

36. Shephard, E., Bedford, R., Milosavljevic, B., Gliga, T., Jones, E. J., Pickles, A., Johnson, M.H., Charman, T., BASIS Team, Baron-Cohen, S. \& Bolton, P. (2018). Early developmental pathways to childhood symptoms of attention-deficit hyperactivity disorder, anxiety and autism spectrum disorder. J Child Psychol Psychiatry.

37. Clifford SM, Hudry K, Elsabbagh M, Charman T, Johnson MH, Team B. Temperament in the first 2 years of life in infants at high-risk for autism spectrum disorders. J Autism Dev Disord. 2013;43(3):673-86. https://doi. org/10.1007/s10803-012-1612-y.

38. Garon N, Zwaigenbaum L, Bryson SE, Smith IM, Brian J, Roncadin C, et al. Temperament and its association with autism symptoms in a high-risk population. J Abnorm Child Psychol. 2016;44(4):757-69. https://doi.org/10.1 007/s10802-015-0064-1.

39. Hendry A, Jones EJ, Bedford R, Gliga T, Charman T, Johnson MH. Developmental change in look durations predicts later effortful control in toddlers at familial risk for ASD. J Neurodev Disord. 2018;10(1):3.

40. Jones EJ, Gliga T, Bedford R, Charman T, Johnson MH. Developmental pathways to autism: a review of prospective studies of infants at risk. Neurosci Biobehav Rev. 2014;39:1-33.

41. Bryson SE, Zwaigenbaum L, McDermott C, Rombough V, Brian J. The autism observation scale for infants: scale development and reliability data. J Autism Dev Disord. 2008;38(4):731-8.

42. Zwaigenbaum L, Bryson S, Rogers T, Roberts W, Brian J, Szatmari P. Behavioral manifestations of autism in the first year of life. Int J Dev Neurosci. 2005;23(2):143-52.

43. Gammer I, Bedford R, Elsabbagh M, Garwood H, Pasco G, Tucker L, et al. Behavioural markers for autism in infancy: scores on the autism observational scale for infants in a prospective study of at-risk siblings. Infant Behav Dev. 2015;38:107-15.

44. Bedford R, Pickles A, Sharp H, Wright N, Hill J. Reduced face preference in infancy: a developmental precursor to callous-unemotional traits? Biol Psychiatry. 2015;78(2):144-50.

45. Mills-Koonce WR, Wagner NJ, Willoughby MT, Stifter C, Blair C, Granger DA, Family Life Project Key Investigators. Greater fear reactivity and psychophysiological hyperactivity among infants with later conduct problems and callous-unemotional traits. J Child Psychol Psychiatry. 2015; 56(2):147-54.

46. Goodman R, Ford T, Richards H, Gatward R, Meltzer H. The development and well-being assessment: description and initial validation of an integrated assessment of child and adolescent psychopathology. J Child Psychol Psychiatry Allied Discip. 2000;41(05):645-55.

47. Rutter M, Bailey A, Lord C. The social communication questionnaire: manual. Los Angeles: Western Psychological Services; 2003.

48. Lord C, Risi S, Lambrecht L, Cook EH Jr, Leventhal BL, DiLavore PC, Rutter M. The Autism Diagnostic Observation Schedule-Generic: a standard measure of social and communication deficits associated with the spectrum of autism. J Autism Dev Disord. 2000;30(3):205-23.

49. Constantino JN. Social Responsiveness Scale, Second Edition (SRS-2). Los Angeles: Western Psychological Services; 2012.

50. Mullen EM. Mullen scales of early learning. Circle Pines: American Guidance Service; 1995.

51. Sparrow SS, Cicchetti DV, Balla DA. Vineland Adaptive Behavior Scales. 2nd ed. Oxford: NCS Pearson Inc.; 2005 
52. Shephard E, Milosavljevic B, Pasco G, Jones EJH, Gliga T, Happe F, Johnson $\mathrm{MH}$, Charman T, The BASIS Team. Mid-childhood outcomes of infant siblings at familial high-risk of autism spectrum disorder. Autism Res. 2017. https://doi.org/10.1002/aur.1733.

53. Lord C, Rutter M, DiLavore P, Risi S, Gotham K, Bishop S. Autism diagnostic observation schedule second edition (ADOS-2) manual (part 1): modules 14. Torrance: Western Psychological Services; 2012.

54. Le Couteur A, Lord C, Rutter M. The Autism Diagnostic Interview-Revised (ADI-R). Los Angeles: Western Psychological Services; 2003.

55. Wechsler D. WASI-II: Wechsler abbreviated scale of intelligence. New York: Psychological Corporation; 2011.

56. Brian J, Bryson SE, Garon N, Roberts W, Smith IM, Szatmari P. Clinical assessment of autism in high-risk 18-month-olds. Autism. 2008;12:433-56.

57. Putnam (2018). Mary Rothbart's Temperament Questionnaires. Retrieved from https://research.bowdoin.edu/rothbart-temperament-questionnaires/ frequently-asked-questions/\#Answer7

58. Conners, C. K. (2008). Conners 3. MHS.

59. Frick PJ. The Inventory of Callous-Unemotional Traits: University of New Orleans; 2004. Unpublished rating scale.

60. StataCorp. Stata Statistical Software: Release13. College Station: StataCorp LP; 2013.

61. Hus V, Bishop S, Gotham K, Huerta M, Lord C. Factors influencing scores on the social responsiveness scale. J Child Psychol Psychiatry. 2013;54(2):216-24.

62. Brian J, Bryson SE, Smith IM, Roberts W, Roncadin C, Szatmari R, Zwaigenbaum L. Stability and change in autism spectrum disorder diagnosis from age 3 to middle childhood in a high-risk sibling cohort. Autism. 2015. https://doi.org/10.1177/1362361315614979 published online ahead of print Dec 15.

63. Bedford R, Gliga T, Shephard E, Elsabbagh M, Pickles A, Charman T, Johnson $\mathrm{MH}$. Neurocognitive and observational markers: prediction of autism spectrum disorder from infancy to mid-childhood. Mol Autism. 2017;8(1):49.

64. Chhabildas N, Pennington BF, Willcutt EG. A comparison of the neuropsychological profiles of the DSM-IV subtypes of ADHD. J Abnorm Child Psychol. 2001;29(6):529-40.

65. Schmitz M, Cadore L, Paczko M, Kipper L, Chaves M, Rohde LA, et al. Neuropsychological performance in DSM-IV ADHD subtypes: an exploratory study with untreated adolescents. Can J Psychiatry. 2002;47(9):863-9.

66. Blair RJR. The amygdala and ventromedial prefrontal cortex in morality and psychopathy. Trends Cogn Sci. 2007;11(9):387-92.

67. Lapierre D, Braun CM, Hodgins S. Ventral frontal deficits in psychopathy: neuropsychological test findings. Neuropsychologia. 1995;33(2):139-51.

68. Ross SR, Benning SD, Adams Z. Symptoms of executive dysfunction are endemic to secondary psychopathy: an examination in criminal offenders and noninstitutionalized young adults. J Personal Disord. 2007;21(4):384-99.

69. Cuthbert BN, Insel TR. Toward the future of psychiatric diagnosis: the seven pillars of RDoC. BMC Med. 2013;11(1):126.

70. Gliga T, Smith TJ, Likely N, Charman T, Johnson MH. Early visual foraging in relationship to familial risk for autism and hyperactivity/inattention. J Atten Disord. 2018;22(9):839-47.

71. Livingston LA, Happé F. Conceptualising compensation in neurodevelopmental disorders: reflections from autism spectrum disorder. Neurosci Biobehav Rev. 2017;80:729-42.

72. Bedford R, Pickles A, Gliga T, Elsabbagh M, Charman T, Johnson MH, BASIS Team. Additive effects of social and non-social attention during infancy relate to later autism spectrum disorder. Dev Sci. 2014;17(4):612-20.

73. Bedford R, Jones E J, Johnson MH, Pickles A, Charman T, Gliga T. Sex differences in the association between infant markers and later autistic traits. Molecular autism. 2016;7(1):21.

74. Weinberg MK, Tronick EZ, Cohn JF, Olson KL. Gender differences in emotional expressivity and self-regulation during early infancy. Dev Psychol. 1999;35(1):175.

75. Gilotty L, Kenworthy L, Sirian L, Black DO, Wagner AE. Adaptive skills and executive function in autism spectrum disorders. Child Neuropsychol. 2002; 8(4):241-8.

76. Clark C, Prior M, Kinsella G. The relationship between executive function abilities, adaptive behaviour, and academic achievement in children with externalising behaviour problems. J Child Psychol Psychiatry. 2002;43:785-96.

77. Diamond A, Lee K. Interventions shown to aid executive function development in children 4 to 12 years old. Science. 2011;333(6045):959-64.

78. Wass SV. Applying cognitive training to target executive functions during early development. Child Neuropsychol. 2015;21(2):150-66.

\section{Publisher's Note}

Springer Nature remains neutral with regard to jurisdictional claims in published maps and institutional affiliations.

\section{Ready to submit your research? Choose BMC and benefit from:}

- fast, convenient online submission

- thorough peer review by experienced researchers in your field

- rapid publication on acceptance

- support for research data, including large and complex data types

- gold Open Access which fosters wider collaboration and increased citations

- maximum visibility for your research: over $100 \mathrm{M}$ website views per year

At BMC, research is always in progress.

Learn more biomedcentral.com/submissions 